\title{
Practice of ARCADIA and Capella in Civil Radar Design
}

\author{
Renfei Xu, Wenhua Fang, and Wei Yin
}

\begin{abstract}
ARChitecture Analysis and Design Integrated Approach (ARCADIA) is a kind of Model-Based System Engineering (MBSE) methodology developed by Thales, and Capella is a kind of modeling tool dedicated to ARCADIA. In this paper, we will introduce our practice of ARCADIA/Capella in civil radar design, including the reason we choose this solution and how we use it in civil radar design. We will also briefly introduce our extension of Capella in parametric modeling, dynamic execution and simulation, system and subsystem collaboration, interface detailed design, and document generation.
\end{abstract}

R. Xu (凶) · W. Fang

Shanghai PGM Technology Co., Ltd., No. 2966 on Jinke Road, Shanghai, China

W. Yin

No. 1th Institute of CETC, No. 8th of Guorui Street, Yuhuatai District, Nanjing, China

(C) The Author(s), under exclusive license to Springer Nature Switzerland AG 2021 